\title{
Hydroxyurea-induced cyclic thrombocytopenia in polycythemia vera
}

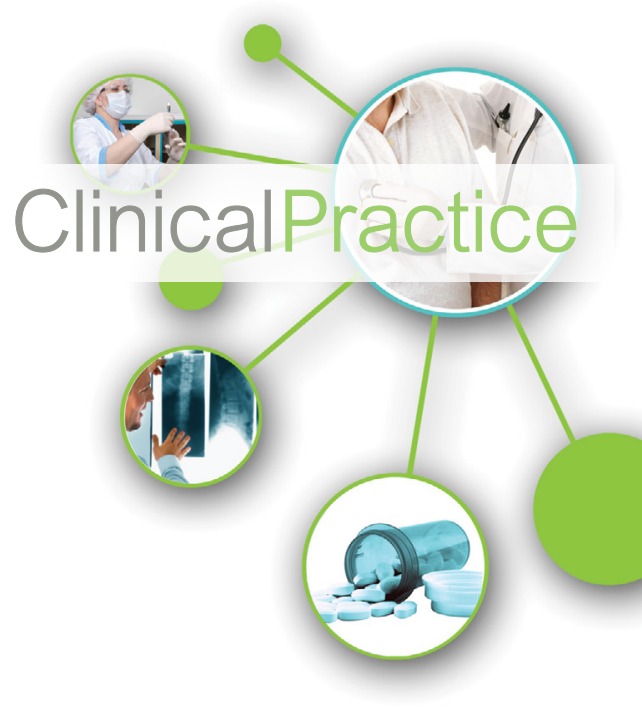

\begin{abstract}
Hydroxyurea is an orally administered medication that is frequently used in the treatment of myeloproliferative disorders as well as sickle cell disease. Cyclical thrombocytopenia during hydroxyurea therapy is an unusual and largely under-recognized side effect. We herein describe a case of a 66-year-old man with polycythemia vera who was started on hydroxyurea, and who subsequently developed platelet count oscillations with peak platelet levels as high as $2900 \times 10^{9} / \mathrm{L}$, and nadirs as low as $8 \times 10^{9} / \mathrm{L}$. We also performed a literature review of the currently published cases of hydroxyurea-induced cyclic thrombocytopenia.
\end{abstract}

Keywords: platelets, thrombocytopenia, hydroxyurea, platelet count oscillation, polycythemia vera

\begin{abstract}
Introduction
Hydroxyurea is an orally administered medication useful in myeloproliferative disorders and sickle cell disease [1,2]. The drug blocks ribonucleotide reductase, inhibiting DNA synthesis and repair. It causes myelosuppression and macrocytosis, and it increases fetal hemoglobin levels $[3,4]$. Unwanted side effects include gastroenterological symptoms, eczema, and distal leg skin ulceration [4]. Cyclic thrombocytopenia is rare, and thus practicing hematologists may be unaware of the phenomenon [5-9]. We herein describe a case that is a helpful reminder of this unusual but important problem, where platelet levels oscillate from thrombocytosis to thrombocytopenic ranges.
\end{abstract}

\section{Case Report}

The patient is a 66-year-old male with a 9-year history of polycythemia vera associated with a jak2 exon 12 mutation (N542-E543 deletion). He presented with RBC $7.44 \times 10^{9} / \mathrm{L}$, hemoglobin $18.6 \mathrm{gm} /$ $\mathrm{dL}$, hematocrit $61.7 \%, \mathrm{MCV} 83 \mathrm{fL}$, platelet count $405 \times 10^{9} / \mathrm{L}$, and WBC $6.3 \times 10^{9} / \mathrm{L}$. Serum erythropoietin level was $2.5 \mathrm{mU} / \mathrm{mL}$ (normal range, $5.0-24.6 \mathrm{mU} / \mathrm{mL}$ ). He was treated with phlebotomies and aspirin 162 mg daily. Hydroxyurea was introduced 1 year after diagnosis, $1.5 \mathrm{gm}$ daily, not adjusted for platelet count.

About a year after hydroxyurea was started, he had a petechial rash on the legs and platelet count $15 \times 10^{9} / \mathrm{L}$. Prednisone was given, and the platelet count improved within days. He was therefore considered to have idiopathic thrombocytopenic purpura (ITP). Thrombocytopenia recurred periodically; the lowest platelet count recorded was $8 \times 10^{9} / \mathrm{L}$, and the highest was $2900 \times 10^{9} / \mathrm{L}$. The WBC remained stable throughout. Rituximab, azathioprine, mycophenolate, and cyclosporin were each briefly tried and were ineffective in preventing platelet count nadirs. The patient experienced neither thromboembolism nor bleeding (apart from petechiae at platelet count nadirs).

In retrospect, predictable cycling was noted with a period of about 28 days, unaffected by "ITP" treatment. Hydroxyurea was discontinued. The platelet count peaked 12 days later at $2907 \times 10^{\%} / \mathrm{L}$ with undetectable serum thrombopoietin (normal range 7-99 $\mathrm{pg} / \mathrm{mL}$ ). Ruxolitinib was introduced and escalated to $20 \mathrm{mg}$ twice daily. In the ensuing 110 days after the peak platelet count, platelet

\section{Karlton Wong* and Michael H Rosove}

University of California, Los Angeles, Department of Medicine, Division of Hematology Oncology, US

*Author for correspondence: KarltonWong@mednet.ucla.edu; Mrosove@mednet.ucla.edu 
counts ranged from $659-1211 \times 10^{9} / \mathrm{L}$ with no regular cycling. Serum thrombopoietin ranged from $49-116 \mathrm{pg} / \mathrm{mL}$.

\section{Discussion and Conclusion}

Tefferi et al. described 2 polycythemia vera patients treated with hydroxyurea who had platelet count oscillations from 35-1412 $\times 10^{9} / \mathrm{L}$ and periods from 27 to 66 days [5]. Serum thrombopoietin levels fluctuated out of phase with platelet counts. Both patients improved with hydroxyurea discontinuation. One had recurrent cycling when rechallenged with the drug.

4 cases were reported by Steensma et al. [6] Platelet counts ranged from 34-1479 $\times 10^{9} / \mathrm{L}$, and cycles lasted 20 to 30 days. One patient had vasomotor symptoms with elevated platelet counts; another developed digital ischemia. In 3 cases, lower peaks and higher nadirs could be achieved by not altering hydroxyurea dosage during oscillations.

Burthem et al. [7] reported a patient with jak2 V617F mutated polycythemia vera in 2008 with platelet count cycling beginning 3 months after hydroxyurea was started. The platelet count range was $200-800 \times 10^{9} / \mathrm{L}$ with a 28-day cycle. Peaks and troughs became less marked when hydroxyurea dosage was not adjusted for the platelet count.

Tauscher et al. [8] reported 12 jak2 V617F mutated polycythemia vera patients who had large platelet count fluctuations during hydroxyurea treatment. One patient's platelet count range was $27-4900 \times 10^{9} / \mathrm{L} ; 6$ patients had nadirs less than $100 \times 10^{9} / \mathrm{L}, 3$ of whom had nadirs less than $50 \times 10^{9} / \mathrm{L}$. Nine patients had peaked over $1000 \times 109 / \mathrm{L}$. Cycling periods ranged from 27 to 30 days. Five patients also had WBC fluctuations with a 26-29 day cycle, counts ranging from 1.9-38.2 × 10\%/L. Most oscillations stopped with hydroxyurea discontinuation; in 2 patients, oscillations gradually disappeared while hydroxyurea was continued. No thromboembolic or bleeding events were reported.

The last report we are aware of was published in 2014, a retrospective analysis of
188 consecutive patients with Philadelphia chromosome-negative myeloproliferative neoplasms [9]. Hydroxyurea was used in 149 patients. Platelet count cycling was defined as a spread between peak and nadir counts greater than $100 \times 10^{9} / \mathrm{L}$. Twenty-nine of the 188 patients $(15.4 \%)$ demonstrated cycling, 25 of whom had received hydroxyurea; 4 had anagrelide. Most oscillations were modest, amplitudes ranging from $100-500 \times 10 \% / \mathrm{L}$ in 23 of 29 patients. Only in 6 were they greater than $500 \times 10^{9} / \mathrm{L}$; 2 were greater than $1200 \times 10^{9} / \mathrm{L}$. The 4 patients with cycling on anagrelide had amplitudes less than $100 \times$ $10^{9} / \mathrm{L}$. Treatment was changed in 7 patients and not changed in 22. No patient had thrombosis or bleeding.

Based on reports in the medical literature coupled with the patient in this report, mild to moderate platelet count oscillations during hydroxyurea therapy for myeloproliferative disorders appear not to be rare, but most cases probably escape notice. Patients with large oscillations appear to be rare but understandably more readily recognized. Patients may be mistakenly diagnosed with ITP as the initiation of ITP therapy is likely to be followed by "response" when it is only a spontaneous upswing of the oscillatory cycle. The commonest cycling period seems to be about 25 to 30 days, which can make it difficult to identify affected patients, especially when peaks and troughs are subtle unless frequent testing is done. Despite extreme platelet count fluctuations in some patients, serious complications appear to be unusual. Withdrawal of hydroxyurea ameliorates extreme cycling. How often stable hydroxyurea dosing might be continued safely requires further clarification. Thus far, the mechanism for cyclic thrombocytopenia remains unclear; however, based on Tefferi's observation that the thrombopoietin level may fluctuate out of phase with the platelet count, [5] as we also observed, hydroxyurea may alter the stability of the thrombopoietin feedback loop, destabilizing the signaling mechanism of the megakaryocyte progenitor pool $[5,10]$. 


\section{References}

Agrawal RK, Patel RK, Shah V, et al. Hydroxyurea in sickle cell disease: drug review. Indian J. Hematol. Blood Transfus. 30(2), 91-96 (2014).

Tefferi A, Barbui T. Polycythemia vera and essential thrombocythemia: 2017 update on diagnosis, risk-stratification, and management. Am. J. Hematol. 92(1), 94-108 (2017).

Yarbro JW. Mechanism of action of hydroxyurea. Semin. Oncol. 1-10 (1992).

Randi ML, Ruzzon E, Tezza F, et al. Toxicity and side effects of hydroxyurea used for primary thrombocytopenia. Platelets. 16(3/4), 181-184 (2005).
Tefferi A, Elliott MA, Kao PC, et al. Hydroxyurea-induced marked oscillations of platelet counts in patients with polycythemia vera. Blood. 96(4), 15821584 (2000).

Steensma DP, Harrison CN, Tefferi A. Hydroxyurea-associated platelet count oscillations in polycythemia vera: A report of four new cases and a review. Leukemia. 42(6), 1243-1253 (2001).

Burthem J, Chaudhry MS. Hydroxycarbamide associated platelet count oscillations in a patient with polycythemia vera. A case report and review of the literature. Platelets. 19(3), 234-235 (2008).
Tauscher J, Siegel F, Petrides PE. Hydroxyurea-induced oscillations in twelve patients with polycythemia vera. Haematological. 95(7), 1227-1229 (2010).

Verner E, Forsyth C, Grigg A. Cyclical thrombocytosis, acquired von Willebrand syndrome and aggressive non-melanoma skin cancers are common in patients with Philadelphia-negative myeloproliferative neoplasms treated with hydroxyurea. Leuk. Lymphoma. 55(5), 1139-1143 (2014).

Santillan M, Mahaffy JM, Belair J, Mackey MC. Regulation of platelet production: the normal response to perturbation and cyclical platelet disease. J. Theor. Biol. 206, 585-603 (2000). 